Research Article

\title{
Power-Minimization-Based Jamming Spectral Design for Finite-Duration Random Target in Clutter
}

\author{
Jing Gao $\mathbb{D}^{1},{ }^{1}$ Rihan $W u\left(\mathbb{D},{ }^{2}\right.$ Xin Guan $\mathbb{D}^{2},{ }^{2}$ Hao Yu $\mathbb{D},{ }^{2}$ and Xishi Wang $\mathbb{D}^{2}$ \\ ${ }^{1}$ School of Control Engineering, Northeastern University at Qinhuangdao, Qinhuangdao 066004, China \\ ${ }^{2}$ School of Computer Science and Engineering, Northeastern University, Shenyang 110819, China \\ Correspondence should be addressed to Jing Gao; summergj@126.com
}

Received 15 January 2021; Revised 10 April 2021; Accepted 23 April 2021; Published 4 May 2021

Academic Editor: Adrian Kliks

Copyright $\odot 2021$ Jing Gao et al. This is an open access article distributed under the Creative Commons Attribution License, which permits unrestricted use, distribution, and reproduction in any medium, provided the original work is properly cited.

\begin{abstract}
An approach to optimize the transmitted waveform of a smart jammer was developed. This study aims to improve power-saving performance by minimizing the total jamming power and allowing the values of signal-to-interference-plus-noise ratio (SINR) and mutual information (MI) between the radar return and target impulse response to be below specific thresholds. First, the model of a finite-duration random target was proposed, and the power-minimization-based optimal jamming waveform design methods in clutter with SINR and MI limitations were investigated. Subsequently, considering the impossibility of obtaining precise prior information for estimation in practice, the spectra are assumed to lie within an uncertainty class where the upper and lower bounds of each frequency point sample are known. Consequently, power-minimization-based single- and double-robust jamming waveform design methods were researched, which bound the worst power-saving performance of the jammer. Finally, we provided several numerical examples to demonstrate that the power-saving performance of smart jammers can be enhanced by employing power-minimization-based optimization algorithms.
\end{abstract}

\section{Introduction}

1.1. Background and Motivation. Advanced signal processing technology intensifies the competition between radars and jammers, which leads to broad research on the interaction between radar and target [1]. As introduced in [2], the signal-to-interference-plus-noise ratio (SINR) and mutual information (MI) between the radar return and target impulse response can be adopted to characterize the detection and parameter estimation performance of the radar system, respectively. Therefore, the performance of the radar can be enhanced by maximizing the SINR or MI values. In [3], Romero et al. proposed a method to optimize matched waveforms for both a known target and random targets, which can be realized by meeting a predefined waveform energy constraint and maximizing the output of SINR and MI. The work in [4] investigated the problem of radar waveform design in tracking parameters of multiple targets, which can be solved by utilizing the information-theoretic water-filling approach. With the requirement of practical application, Ahmad and Amin proposed a problem of matched illumination waveform design to improve the performance of target detection, which can be applied to through-the-wall radar imaging and sensing applications [5]. As introduced in [6], waveform design algorithms for multiple-input multiple-output (MIMO) radar are presented in colored noise, which considers the issue of MI and the relative entropy maximization. A low probability of intercept (LPI)-based orthogonal frequency division multiplexing (OFDM) modulation waveform design method is proposed, which can enhance the LPI performance for an integrated radar and communications system [7]. Cheng et al. studied the robust joint design of the transmitted waveform and filtering structure, where the output of the SINR is considered to be in the worst-case scenario [8]. In addition, the method of signal processing based on subarrayed frequency diverse array is investigated to counteract deceptive electronic countermeasure signals [9]. Moreover, Ciuonzo et al. solved the issue of intrapulse radar-embedded communication and proposed a novel waveform design 
scheme based on a multiobjective optimization paradigm [10].

However, all the studies proposed above prefer to enhance the performance of target detection and parameter estimation without considering the existence of jamming equipment. In effect, the anti-jamming performance of radar should also be taken seriously. Because the anti-jamming performance of radar can be improved by advanced waveforms, many schemes have been studied for the improvement of anti-interference. For instance, reference [11] proposed a method of interference suppression, which mainly adopts waveform watermarking technology. Further, the work in [12] researched the problem of waveform design for lightening single-tone jamming (STJ) signals in time-hopping spread spectrum (TH SS) systems, which enhances the anti-jamming performance of TH SS systems. In addition, the radar-transmitted waveform and mismatch filter are jointly designed to suppress the interrupted sampling repeater jamming (ISRJ) [13].

1.2. Related Work. Motivated by the development of interference suppression technology, a better jamming waveform design scheme is needed for self-preservation. For instance, in [14], the methods of waveform design of smart jammers are investigated based on SINR and MI to interfere with the target detection and weaken the estimation performance of the radar. In addition, based on the imprecision of spectrum estimation, the work in [15] researched the minimax robust jamming techniques based on SINR and MI, where the worst-case jamming performance is enhanced. Subsequently, the jamming power allocation techniques for MIMO radar were researched based on the minimum mean square error (MMSE) and MI [16]. However, all the schemes introduced above tend to improve the jamming performance without considering the low probability of intercept (LPI) of the smart jammer. Consequently, the LPI-based adaptive jamming waveform design methods for distributed multiple-radar were investigated in [17]. The obtained algorithms are beneficial for the development of smart jammers. However, spectrum estimation ability is limited. Estimation and analysis of spectrum parameters are imprecise; therefore, the model of uncertainty class was designed in [18], where the target power spectral density (PSD) is assumed to lie within an uncertainty range. Based on this uncertainty model, robust waveform design methods have been extensively researched [19-21]. In particular, literature [22] proposes three different robust radar-transmitted waveforms, which minimize the worst-case radartransmitted power in three different cases.

In conclusion, the works described above show that the uncertainty model is extensively applied in waveform design research. Although robust jamming waveform design approaches with an estimated radar-transmitted waveform is provided, these methods assume that the target PSD obtained by the jammer is precise. In addition, for the presence of a finite-duration random target, we noted that the powerminimization-based jamming waveform design with an estimated radar-transmitted waveform and target PSD has not been studied. Consequently, the problems of power- minimization-based single-robust and double-robust jamming waveform design methods with SINR and MI criteria for random targets are investigated.

1.3. Major Contributions. Motivated by the aforementioned facts, we present power-minimization-based adaptive jamming waveform design methods for finite-duration random target to adapt to a complex electromagnetic environment. Our previous research [23] was the first to investigate a robust jamming waveform design based on power minimization. Moreover, this study extends the content to three aspects. First, the existence of clutter was considered in the optimal jamming waveform design. Second, the target model is extended from a deterministic model to a stochastic target with a finite duration. Third, the power-saving performance of the wideband jamming waveform was simulated for comparison. The main contributions of this study are summarized as follows:

(1) An optimal jamming waveform design method for a finite-duration target in clutter was proposed. After establishing the signal model of the finite-duration random target in signal-dependent interference, a mathematical model based on power minimization was designed. By minimizing the total transmitted power of the smart jammer and making the SINR or MI value less than a specific threshold, the adaptive jamming spectrum maximizes its power-saving performance and limits the ability of target detection and parameter estimation of radar.

(2) The research above assumes that the radar waveform has been intercepted by the reconnaissance system; therefore, the parameters of the radar-transmitted spectrum can be estimated precisely. However, a perfect estimation of the radar signal is practically impossible. Because the radar-transmitted spectrum obtained by the smart jammer is imprecise, the power-minimization-based single-robust jamming waveforms with SINR and MI criteria for finiteduration random target are designed, which can finally optimize the power-saving performance of smart jammers in the local-worst case.

(3) A common assumption in the literature of jamming waveform design is that the jammer has all the parameters of the target spectrum. However, based on the fact that some jammers such as ground jammers are not integrated with the target, it is necessary to consider the inaccuracy of the target spectrum obtained by the jammer. Therefore, the power-minimization-based double-robust jamming waveforms with SINR and MI criteria for finiteduration random targets are designed, which can minimize the power consumption of the smart jammer in the global-worst case.

1.4. Organization. The remainder of this paper is organized as follows. In Section 2, the signal model of a finite-duration random target in signal-dependent interference is established. 
The power-minimization-based optimal jamming waveform design with SINR and MI criteria in clutter is described in Section 3. Based on the uncertainty range of the prior information, Section 4 proposes power-minimization-based singlerobust and double-robust jamming spectra to optimize the worst-case performance. Section 5 presents the simulation results of the three different jamming spectra and provides the corresponding analysis. Finally, the conclusions are presented in Section 6.

The notations used in this study are as follows: the continuous time-domain signal is denoted by $x(t)$, the Fourier transform of $x(t)$ is $X(f)$, the symbol * denotes the convolution operator, and $E[\cdot]$ denotes the expectation of an input entity.

\section{System and Signal Models}

2.1. System Model. As depicted in Figure 1, let us consider a general jamming scenario, where a radar is used to detect a random target or estimate the target parameters. The radar transmits the signal adaptively according to the parameters of the echoes scattered off the target. The smart jammer disturbs the radar and completes specific jamming tasks. Clutter is a signal-dependent interference component, e.g., ground clutter.

The major objective of smart jammers is to maximize the power-saving performance, while limiting the detection and parameter estimation performance of radar systems. Because the ability of jammers to obtain prior information is different, three situations are considered. (1) The precise radar-transmitted waveform and target PSD can be intercepted by a smart jammer. (2) The radar-transmitted waveform obtained by the jammer is fuzzy. (3) Both the radar-transmitted waveform and target PSD are estimated by the jammer and are all imprecise. In Figure 1, $x(t)$ is the radar-transmitted waveform and $j(t)$ is the jamming signal launched by the smart jammer.

2.2. Signal Model for Finite-Duration Stochastic Target. As opposed to a known target and an infinite-time random target, it is assumed that the target is a finite-duration process. The problem of jamming design can be investigated for a scenario with this type of target. The realization of such a stochastic process is shown in Figure 2(a), where $g(t)$ is a complex wide-sense stationary process with some PSD and $a(t)$ is a rectangular window function of duration $T_{h}$. It can be noted that the product $h(t)=a(t) g(t)$ denotes complexvalued baseband target impulse response supported only in the range of $\left[0, T_{h}\right]$. In the case where $g(t)$ is a wide-sense stationary process, $\mathbf{h}(t)$ is locally stationary within $\left[0, T_{h}\right]$.

The signal model of a finite-duration random target with a jammer in clutter is displayed in Figure 2(b), where $h(t)$ and $x(t)$ denote a finite-duration random target of duration $T_{h}$ and the complex-valued baseband transmit waveform, respectively. The Fourier transforms of $x(t)$ and $h(t)$ can be denoted by $X(f)$ and $H(f)$, respectively. As an interference component, $c(t)$ is a complex-valued, zero-mean Gaussian random process, which has the power spectral density (PSD) represented as $S_{\mathrm{cc}}(f)$. Similarly, $j(t)$ is a complex-valued, zero-mean Gaussian random process with the PSD $J(f)$. Let

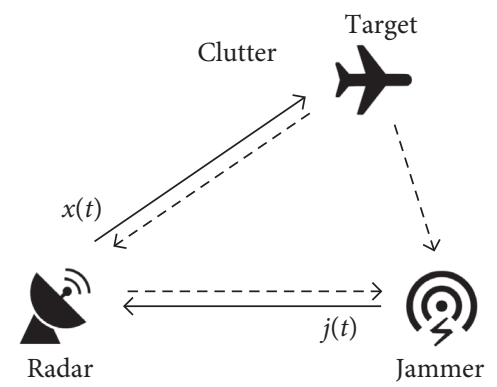

Figure 1: System model.

$n(t)$ be a complex-valued, zero-mean channel noise process with the PSD $S_{\mathrm{nn}}(f) . r(t)$ represents the complex-valued receiver-filter impulse response. As shown in Figure 2(b), the output signal of the receiver filter is $y(t)$, which can be expressed as follows [3]:

$$
y(t)=r(t) *[x(t) * h(t)+x(t) * c(t)+j(t)+n(t)] .
$$

The symbol * denotes the convolution operator. The received signal of the radar system and noise components can be expressed by

$$
\begin{aligned}
& y_{s}(t)=r(t) * x(t) * h(t), \\
& y_{n}(t)=r(t) *[x(t) * c(t)+j(t)+n(t)],
\end{aligned}
$$

respectively.

The energy spectrum variance (ESV) of $h(t)$ can be represented as [3]

$$
\sigma_{H}^{2}(f)=E\left[\left|H(f)-\mu_{H}(f)\right|^{2}\right]
$$

where $\mu_{H}(f)$ denotes the mean of $H(f)$, which was assumed to be zero. The power spectrum variance (PSV) of $h(t)$ can be expressed as follows:

$$
\gamma_{H}^{2}(f)=\frac{\sigma_{H}^{2}(f)}{T_{h}}=\frac{E\left[|H(f)|^{2}\right]}{T_{h}} .
$$

The SINR expression for the finite-duration random target can be defined as follows [15]:

$$
\operatorname{SINR}=T_{y} \int_{-\infty}^{\infty} \frac{\alpha \gamma_{H}^{2}(f)|X(f)|^{2}}{S_{\mathrm{cc}}(f)|X(f)|^{2}+J(f)+S_{\mathrm{nn}}(f)} \mathrm{d} f,
$$

where $\alpha=T_{h} / T_{y}, T_{y}$ represents the duration of $y(t), \alpha$ indicates that the convolution output of $H(f)$, and $X(f)$ is only stationary for a finite time window. Hence, we can define the SINR spectral density as follows:

$$
R_{\mathrm{SINR}}=\frac{\alpha \gamma_{H}^{2}(f)|X(f)|^{2}}{S_{\mathrm{cc}}(f)|X(f)|^{2}+J(f)+S_{\mathrm{nn}}(f)} .
$$

The waveform energy is concentrated in the BW band, and the SINR expression can be simplified as follows:

$$
\operatorname{SINR}=\int_{\mathrm{BW}} \frac{\sigma_{H}^{2}(f)|X(f)|^{2}}{S_{\mathrm{cc}}(f)|X(f)|^{2}+S_{\mathrm{nn}}(f)+J(f)} \mathrm{d} f .
$$




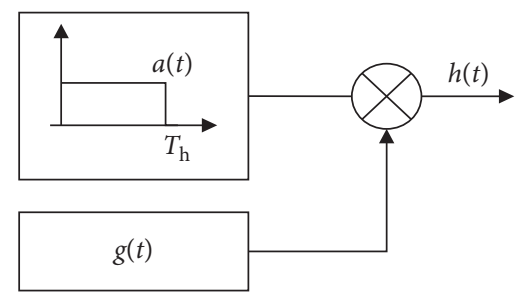

(a)

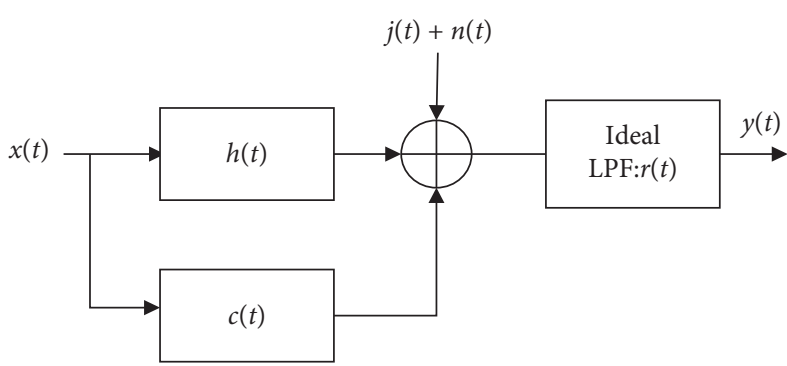

(b)

Figure 2: Signal model (a) for finite-duration random target and (b) for jamming spectral design.

Similarly, the overall MI can be defined as follows [3]:

$$
\begin{aligned}
\mathrm{MI} & =I(y(t) ; h(t) \mid x(t)) \\
& =\operatorname{Ty} \int_{\mathrm{BW}} \ln \left(1+\frac{\sigma_{H}^{2}(f)|X(f)|^{2}}{\operatorname{Ty}\left(|X(f)|^{2} S_{\mathrm{cc}}(f)+J(f)+S_{\mathrm{nn}}(f)\right)}\right) \mathrm{d} f \\
& =\operatorname{Ty} \int_{\mathrm{BW}} \ln \left(1+\alpha\left\{\frac{\gamma_{H}^{2}(f)|X(f)|^{2}}{|X(f)|^{2} S_{\mathrm{cc}}(f)+J(f)+S_{\mathrm{nn}}(f)}\right\}\right) \mathrm{d} f .
\end{aligned}
$$

\section{Optimal Jamming Spectral Design}

In this section, the power-minimization-based jamming design method is proposed to limit the detection and parameter estimation performance of the radar system, while minimizing the total jamming power. As mentioned above, based on the different abilities of the jammer to obtain the prior information, three situations are considered. First, it can be assumed that the precise characteristics of the radartransmitted waveform and target PSD can be perfectly estimated using a smart jammer. Thus, as a result of precise prior information, an optimal jamming waveform can be obtained.

As mentioned above, the primary purpose of this optimization scheme is to minimize the power consumption of smart jammer, while limiting the detection and parameter estimation performance of the radar system. Moreover, as shown in [2], the SINR and MI can be utilized as metrics for the target characterization and parameter estimation performance of the radar system. Therefore, this optimization scheme can be summarized as a problem of designing a strategy to minimize the jammer transmitted power and letting the value of SINR and MI be below a specified threshold. The scheme can be solved using the Lagrange multiplier theory. The specific analyses were as follows.

\subsection{Power-Minimization-Based Jamming Spectrum Design} with SINR Limitation. Based on the derivation in Section 2, the expression of the SINR is shown in (7). Evidently, the value of SINR is related to the target PSV, radar-transmitted waveform, noise PSD, clutter PSD, and jamming PSD. The aim of the smart jammer is to prevent radar from being detected. Therefore, we should degrade the SINR performance for fixed parameters of the target spectrum, radar-transmitted waveform, the noise PSD, and the clutter PSD. A higher jamming power can degrade the detection performance of radar system. Thus, the value of SINR should be below a specified level, which can be determined by the requirement for the jamming task.

To weaken the detection ability of the radar system and guarantee optimal power-saving performance, a mathematical model can be established, which minimizes the total jamming power and makes the output SINR less than a specified threshold. Finally, we can design the minimization problem as

$$
\begin{aligned}
& \min \int_{\mathrm{BW}} J(f) \mathrm{d} f \\
& \text { s.t. } \int_{\mathrm{BW}} \frac{\sigma_{H}^{2}(f)|X(f)|^{2}}{V(f)+J(f)} \mathrm{d} f \leq \gamma_{\mathrm{SINR}},
\end{aligned}
$$

where $\gamma_{\text {SINR }}$ denotes the SINR threshold, which is determined by jamming task. The optimal solution is mathematically represented as follows:

$$
J(f)=\max [0, B(f)(A-D(f))]
$$

where $B(f)$ and $D(f)$ are

$$
\begin{aligned}
& B(f)=|X(f)| \sqrt{\sigma_{H}^{2}(f)}, \\
& D(f)=\frac{V(f)}{|X(f)| \sqrt{\sigma_{H}^{2}(f)}},
\end{aligned}
$$

where $V(f)$ can be denoted as follows:

$$
V(f)=S_{\mathrm{cc}}(f)|X(f)|^{2}+S_{\mathrm{nn}}(f) .
$$

Considering the SINR threshold constraint, $A$ is a constant that can be determined by the following equation:

$$
\int_{\text {BW }} \frac{|X(f)|^{2} \sigma_{H}^{2}(f)}{\max [0, B(f)(A-D(f))]+V(f)} \mathrm{d} f \leq \gamma_{\mathrm{SINR}} .
$$

This optimization problem can be solved by employing the Lagrange multipliers, and the analytical solution of the jamming waveform with the SINR criterion is derived in Appendix A. 
3.2. Power-Minimization-Based Jamming Spectrum Design with MI Limitation. According to the derivation in Section 2, the expression of MI is shown in (8). The MI is related to the target ESV, radar-transmitted waveform, noise PSD, clutter PSD, and jamming PSD. The aim of a smart jammer is to prevent the radar from estimating the parameter of the random target. Therefore, we should degrade the MI performance for fixed parameters of the target spectrum, the radar-transmitted waveform, the noise PSD, and the clutter PSD. Evidently, a higher jamming power can degrade the parameter estimation performance of the radar. Therefore, the achieved MI should be below a specified level, which can be determined by the requirement for the jamming task.

To weaken the parameter estimation performance of the radar system and guarantee optimal power-saving performance of smart jammer, a mathematical model can be established, which minimizes the total jamming power and makes the MI less than a specified threshold. The mathematical model can be denoted as follows:

$$
\begin{aligned}
& \min \int_{\mathrm{BW}} J^{*}(f) \mathrm{d} f \\
& \text { s.t. } \operatorname{Ty} \int_{\mathrm{BW}} \ln \left(1+\frac{\sigma_{H}^{2}(f)|X(f)|^{2}}{\operatorname{Ty}\left(V(f)+S_{\mathrm{nn}}(f)\right)}\right) \mathrm{d} f \leq \gamma_{\mathrm{MI}},
\end{aligned}
$$

where $\gamma_{\mathrm{MI}}$ indicates the MI threshold, which can be determined by jamming task. The optimal jamming spectrum satisfying the mathematical model (14) can be expressed as follows:

$$
J^{*}(f)=\max \left[0, G(f)\left(A^{*}-C(f)\right)\right]
$$

where

$$
\begin{aligned}
G(f) & =\frac{\left(\sigma_{H}^{2}(f)|X(f)|^{2}\right) / \mathrm{Ty}}{\left(2 V(f)+\sigma_{H}^{2}(f)|X(f)|^{2}\right) / \mathrm{Ty}}, \\
C(f) & =\frac{\left(V^{2}(f)+\sigma_{H}^{2}(f)|X(f)|^{2} V(f)\right) / \mathrm{Ty}}{\left(\sigma_{H}^{2}(f)|X(f)|^{2}\right) / \mathrm{Ty}} .
\end{aligned}
$$

Considering the MI threshold constraint, $A^{*}$ is a constant and can be defined by the MI threshold constraint.

$$
\operatorname{Ty} \int_{\mathrm{BW}} \ln \left(1+\frac{\sigma_{H}^{2}(f)|X(f)|^{2}}{\operatorname{Ty}\left(\max \left[0, G(f)\left(A^{*}-C(f)\right)\right]+V(f)\right)}\right) \mathrm{d} f \leq \gamma_{\mathrm{MI}} .
$$

This optimization problem can be solved by employing the Lagrange multipliers, and the analytical solution of the jamming waveform with the MI criterion is derived in Appendix B.

\section{Robust Jamming Spectral Design}

In this section, based on the fact that the abilities of jammers to obtain prior information are different, two more cases are considered. First, it can be assumed that the radar-transmitted waveform obtained by the jammer is fuzzy. Alternatively, the target PSD can be perfectly estimated using a smart jammer. Subsequently, the worst case was considered. Both the radar-transmitted waveform and target PSD are estimated by a jammer and are all imprecise. Based on these two situations, power-minimization-based single-robust and double-robust jamming waveform design methods are investigated. Significantly, the impact of clutter on the radar system is ignored to simplify the scheme in this section.

4.1. Power-Minimization-Based Single-Robust Jamming Spectra Design. In this subsection, the spectral uncertainty of the radar-transmitted waveform is considered, and the band model in [18] is employed. The spectral uncertainty range of the radar-transmitted waveform is assumed to lie within an uncertainty class where the upper and lower bounds of each frequency point sample are known, which can be represented as follows:

$$
\begin{aligned}
X(f) & \in \tau \\
& =\left\{l_{k} \leq\left|X\left(f_{k}\right)\right| \leq u_{k}, \quad \text { for } k=1,2,3, \ldots, K\right\},
\end{aligned}
$$

where the frequency samples are represented by $f_{k}$ and the band model of [16] is employed in Figure 3. This model is widely applied in robust signal processing [15] because the confidence band can be defined via spectrum estimation. The solid line denotes the real radar waveform. The range between each frequency point of this band model changes randomly. A larger range between the upper and lower bounds indicates greater uncertainty, and vice versa.

For each specific spectrum in the uncertainty class, an optimal jamming waveform was generated. To ensure the worst-case performance of a smart jammer, robust jamming waveform design methods were proposed. Based on the different jamming tasks, single-robust jamming waveform design techniques based on SINR and MI are introduced.

4.1.1. Single-Robust Jamming Spectrum Design with SINR Limitation. As previously mentioned, it is assumed that the impact of clutter on the radar system is ignored to simplify the scheme. The relationship between the output SINR and $X(f)$ is given by the following equation:

$$
\operatorname{SINR}=\int_{\mathrm{BW}} \frac{\sigma_{H}^{2}(f)|X(f)|^{2}}{J(f)+S_{\mathrm{nn}}(f)} \mathrm{d} f .
$$

Evidently, the jammer has the worst performance when $X^{\text {worst }}(f)=U(f)$ is adopted, and the SINR value is maximum at this time, which can be denoted by (20). $X^{\text {worst }}(f)$ represents the radar waveform when the jammer is the worst-case scenario. $U(f)$ denotes the upper bound of the uncertainty class $\tau$.

$$
\operatorname{SINR}^{\max }=\int_{\mathrm{BW}} \frac{\sigma_{H}^{2}(f)|U(f)|^{2}}{J(f)+S_{\mathrm{nn}}(f)} \mathrm{d} f .
$$

Thus, the power-minimization-based single-robust jamming waveform design with the SINR criterion can be denoted as follows: 


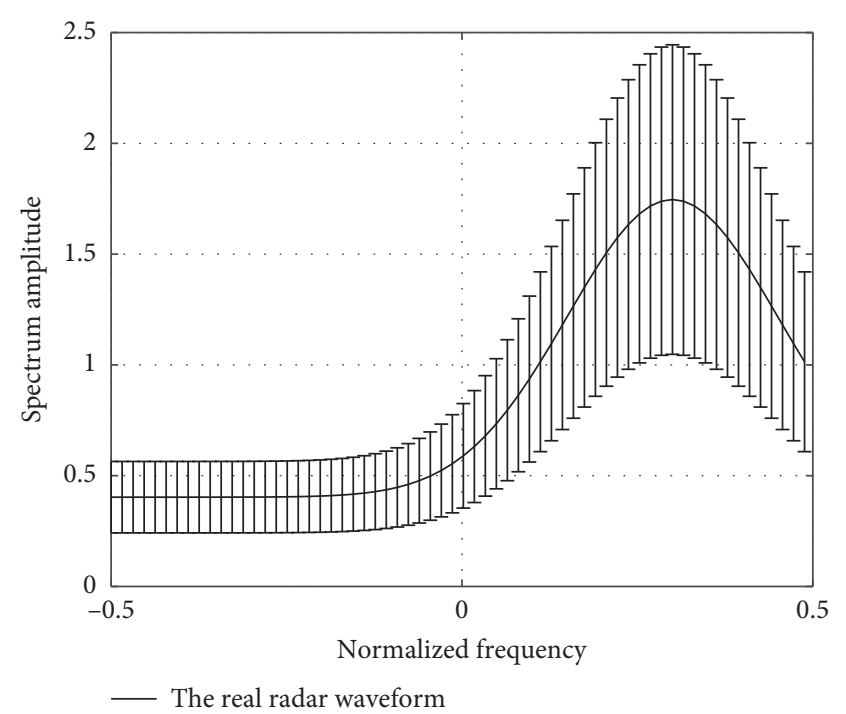

FIgURE 3: The uncertainty class of the radar waveform.

$$
\begin{aligned}
& \min \int_{\mathrm{BW}}{ }_{\text {robust }}^{\text {roux }}(f) \mathrm{d} f \\
& \text { s.t. } \mathrm{SINR}^{\max } \leq \gamma_{\mathrm{SINR}},
\end{aligned}
$$

where $|U(f)|=\left\{u_{k}, k=1, \ldots, K\right\}$. The optimal jamming waveform that minimizes the total jamming power with the SINR limitation can be expressed as (22), which can be derived as in Appendix A:

$$
J_{\text {robust }}^{-}(f)=\max [0, B \overline{(f)}(\bar{A}-\bar{D}(f))],
$$

where $B \overline{(f)}$ and $\bar{D}(f)$ are as follows:

$$
\begin{aligned}
& B \overline{(f)}=|U(f)| \sqrt{\sigma_{H}^{2}(f)}, \\
& \bar{D}(f)=\frac{S_{\mathrm{nn}}(f)}{|U(f)| \sqrt{\sigma_{H}^{2}(f)}}
\end{aligned}
$$

Considering the SINR threshold constraint, $\bar{A}$ is a constant that can be determined by the following equation:

$$
\int \frac{\sigma_{H}^{2}(f)|U(f)|^{2}}{{ }_{\mathrm{BW}} \max [0, B(f)(\bar{A}-\bar{D}(f))]+S_{\mathrm{nn}}(f)} \mathrm{d} f \leq \gamma_{\mathrm{SINR}} .
$$

4.1.2. Single-Robust Jamming Spectrum Design with MI Limitation. The relationship between the output MI and $X(f)$ is shown in (25) with a neglected clutter.

$$
\begin{aligned}
\mathrm{MI} & =I(y(t) ; h(t) \mid x(t)) \\
& =\mathrm{Ty} \int_{\mathrm{BW}} \ln \left(1+\frac{\sigma_{H}^{2}(f)|X(f)|^{2}}{\mathrm{Ty}\left(J(f)+S_{\mathrm{nn}}(f)\right)}\right) \mathrm{d} f .
\end{aligned}
$$

Evidently, the jammer has the worst performance when $X^{\text {worst }}(f)=U(f)$ is adopted. $X^{\text {worst }}(f)$ represents the radar waveform when the jammer is the worst-case scenario. $U(f)$ denotes the upper bound of the uncertainty class $\tau$. The maximum value of MI can be expressed as follows:

$$
\mathrm{MI}^{\max }=\operatorname{Ty} \int_{\mathrm{BW}} \ln \left(1+\frac{\sigma_{H}^{2}(f)|U(f)|^{2}}{\mathrm{Ty}\left(J(f)+S_{\mathrm{nn}}(f)\right)}\right) \mathrm{d} f .
$$

Thus, the power-minimization-based single-robust jamming waveform design with the MI criterion can be denoted as (27), which can be derived as in Appendix B:

$$
\begin{aligned}
& \min \int_{\mathrm{BW}} J_{\text {robust }}^{-}(f) \mathrm{d} f \\
& \text { s.t. } \mathrm{MI}^{\max } \leq \gamma_{\mathrm{MI}},
\end{aligned}
$$

where $|U(f)|=\left\{u_{k}, k=1, \ldots, K\right\}$. The optimal jamming waveform that minimizes the total jamming power with the MI limitation can be denoted as follows:

$$
\begin{aligned}
J_{\text {robust* }}(f) & =\max \left[0, G \overline{(f)}\left(\bar{A}^{*}-C \overline{(f)}\right)\right], \\
\bar{G}(f) & =\frac{\left(\sigma_{H}^{2}(f)|U(f)|^{2}\right) / \mathrm{Ty}}{\left(2 S_{\mathrm{nn}}(f)+\sigma_{H}^{2}(f)|U(f)|^{2}\right) / \mathrm{Ty}},
\end{aligned}
$$

where $\bar{A}^{*}$ is a constant that can be defined by the MI threshold constraint.

$$
\operatorname{Ty} \int_{\mathrm{BW}} \ln \left(1+\frac{\sigma_{H}^{2}(f)|U(f)|^{2}}{\operatorname{Ty}\left(\max \left[0, \overline{(f)}\left(\bar{A}^{*}-\overline{(f)}\right)\right]+S_{n n}(f)\right)}\right) \mathrm{d} f \leq \gamma_{\mathrm{MI}} \text {. }
$$

4.2. Power-Minimization-Based Double-Robust Jamming Spectra Design. Based on the power-minimization-based single-robust jamming waveform design method, the spectral uncertainty of the target spectrum is considered in this subsection, and the band model in [18] is employed again. The spectrum uncertainty range of the target spectrum is assumed to lie within an uncertainty class, where the upper and lower bounds of each frequency point sample are known, which can be represented as follows: 


$$
\begin{aligned}
& H(f) \in v \\
& =\left\{d_{k} \leq\left|H\left(f_{k}\right)\right| \leq s_{k}, \quad \text { for } k=1,2,3, \ldots, K\right\},
\end{aligned}
$$

where the frequency samples are still denoted by $f_{k}$ and the band model of [16] is employed in Figure 4. The solid line denotes a real random target. Likewise, the range between each frequency point of this band model changes randomly. A larger range between the upper and lower bounds indicates greater uncertainty, and vice versa.

For each specific spectrum in the uncertainty class, an optimal jamming waveform was generated. To ensure the global-worst case performance of the smart jammer, doublerobust jamming waveform design methods were investigated. Based on the different jamming tasks, double-robust jamming waveform design techniques with SINR and MI criteria are introduced. In this section, the impact of clutter on radar systems is ignored to simplify the scheme.

\subsubsection{Double-Robust Jamming Spectrum Design with SINR} Limitation. The relation between the value of the SINR and $X(f)$ is expressed in (19). Evidently, the jammer has the worst performance when $X^{\text {worst }}(f)=U(f)$ and $H^{\text {worst }}(f)=$ $S(f)$ are adopted, and the SINR value is maximum at this time, which can be denoted by (31). $X^{\text {worst }}(f)$ and $H^{\text {worst }}(f)$ represent the radar waveform and target spectrum when the jammer is at the global-worst case. $U(f)$ and $S(f)$ denote the upper bounds of the uncertainty class $\tau$ and $v$, respectively.

$$
\operatorname{SINR}^{\text {maximum }}=\int_{\mathrm{BW}} \frac{\sigma_{S}^{2}(f)|U(f)|^{2}}{J(f)+S_{\mathrm{nn}}(f)} \mathrm{d} f .
$$

Thus, the power-minimization-based double-robust jamming spectrum design with SINR limitation can be denoted as follows:

$$
\begin{aligned}
& \min \int_{\mathrm{BW}} \underset{\text { robust }}{=}(f) \mathrm{d} f \\
& \text { s.t. } \operatorname{SINR}^{\text {maximum }} \leq \gamma_{\mathrm{SINR}},
\end{aligned}
$$

where $|U(f)|=\left\{u_{k}, k=1, \ldots, K\right\} \quad$ and $\quad|S(f)|=\left\{s_{k}\right.$, $k=1, \ldots, K\}$. The jamming spectrum with estimated spectra of radar and target minimizing the total jamming power with SINR limitation can be indicated as (33), which can be derived as similar in Appendix A:

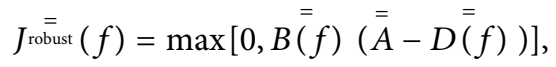

where $B \stackrel{\overline{(}}{f})$ and $D \stackrel{\overline{(}}{f})$ are as follows:

$$
\begin{aligned}
& B \stackrel{\overline{(} f)}{=}=|U(f)| \sqrt{\sigma_{S}^{2}(f)}, \\
& \dot{\overline{(} f} f)=\frac{S_{\mathrm{nn}}(f)}{|U(f)| \sqrt{\sigma_{S}^{2}(f)}},
\end{aligned}
$$

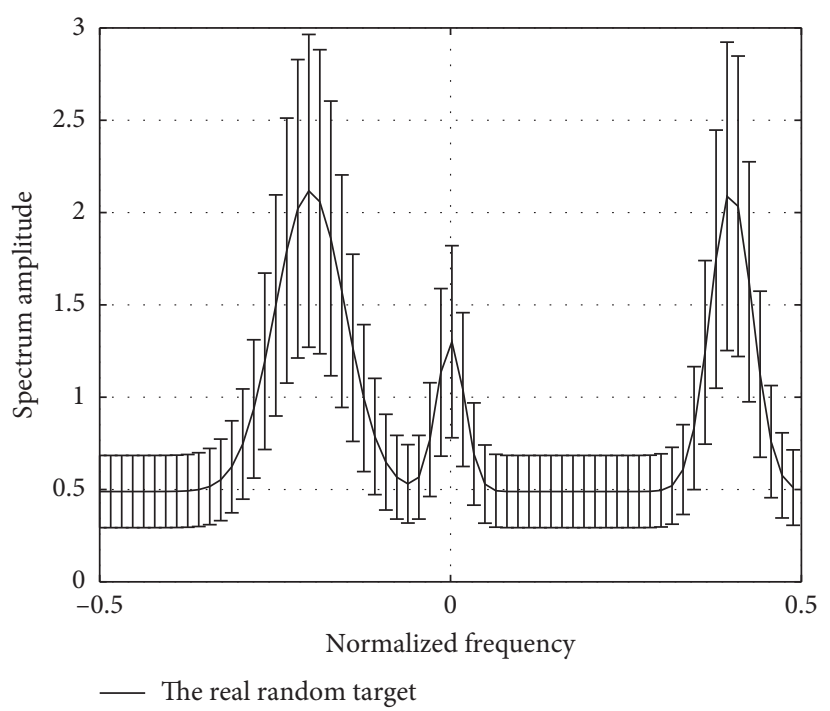

Figure 4: The uncertainty class of the target spectrum.

respectively.

$A$ is a constant that can be defined by the SINR threshold constraint.

$$
\int_{\mathrm{BW}} \frac{\sigma_{S}^{2}(f)|U(f)|^{2}}{=\overline{m a x}[0, B(f)(\stackrel{=}{=}-D(f))]+S_{\mathrm{nn}}(f)} \mathrm{d} f \leq \gamma_{\mathrm{SINR}} .
$$

4.2.2. Double-Robust Jamming Spectrum Design with MI Criterion. The relation between the MI value and $X(f)$ is shown in (25) with neglected clutter. Obviously, the jammer has the worst performance when $X^{\text {worst }}(f)=U(f)$ and $H^{\text {worst }}(f)=S(f)$ are adopted, and the MI value is maximum at this time, which can be denoted by the following equation:

$$
\mathrm{MI}^{\text {maximum }}=\mathrm{Ty} \int_{\mathrm{BW}} \ln \left(1+\frac{\sigma_{S}^{2}(f)|U(f)|^{2}}{\mathrm{Ty}\left(J(f)+S_{\mathrm{nn}}(f)\right)}\right) \mathrm{d} f .
$$

Thus, the power-minimization-based double-robust jamming spectral design with the MI limitation can be indicated as (37), which can be derived as similar as Appendix B:

$$
\begin{aligned}
& \min \int_{\mathrm{BW}} \underset{\text { robust * }}{=}(f) \mathrm{d} f, \\
& \text { s.t. } \mathrm{MI}^{\text {maximum }} \leq \gamma_{\mathrm{MI}} .
\end{aligned}
$$

The double-robust jamming spectrum minimizing the total jamming power with MI limitation can be expressed as follows: 


$$
\begin{aligned}
& \underset{J^{\text {robust * }}}{=}(f)=\max \left[0, \stackrel{=}{(f)}\left(\stackrel{=}{A^{*}}-C \stackrel{\overline{(}}{(f)}\right)\right] \text {, } \\
& \underset{\overline{(}}{(f)}=\frac{\left(\sigma_{S}^{2}(f)|U(f)|^{2}\right) / \mathrm{Ty}}{\left(2 S_{\mathrm{nn}}(f)+\sigma_{S}^{2}(f)|U(f)|^{2}\right) / \mathrm{Ty}},
\end{aligned}
$$

where $\bar{A}^{*}$ is a constant that can be determined by the MI threshold constraint.

$$
\operatorname{Ty} \int_{\mathrm{BW}} \ln \left(1+\frac{\sigma_{S}^{2}(f)|U(f)|^{2}}{\operatorname{Ty}\left(\max \left[0, \underset{=}{(f)}\left(\stackrel{\bar{\prime}}{A^{*}-C(f)}\right)\right]+S_{\mathrm{nn}}(f)\right)}\right) \mathrm{d} f \leq \gamma_{\mathrm{MI}}
$$

\section{Simulation and Results}

In this section, we provide several numerical examples to demonstrate the accuracy of the proposed jamming waveform design method and the improvement of the powersaving performance of the optimization scheme.

5.1. Simulation Parameters. The default parameters for all the experiments are provided in Table 1. Without loss of generality, we employed specific spectra as prior information in the environment. Figure 5 shows the target spectrum, the PSD of noise, and the radar-transmitted waveform, which can be estimated by the smart jammer. As mentioned above, power-minimization-based jamming waveform design methods are applied to finite-duration random targets. Therefore, three random target spectra were generated to verify the effectiveness of this algorithm, as shown in Figure 6.

The uncertainty range of the radar waveform is shown in Figure 7. The spectral amplitude between the upper and lower bounds of each frequency point is different, and the upper and lower bounds are identified by the short horizontal line. Similarly, Figure 8 indicates the spectrum uncertainty range for the finite-duration target 1 . The uncertainty ranges of the other target spectra are similar to those in Figure 8, which are not illustrated for clarification.

5.2. Simulation Results. In this subsection, power-minimization-based jamming spectra were designed to limit the detection and parameter estimation performance of the radar system. Corresponding to the three cases mentioned above, optimal, single-robust, and double-robust jamming spectra were designed. First, optimal jamming spectra with SINR and MI criteria were obtained for precise radartransmitted waveforms and target spectrum, as depicted in Figures 5 and 6. Subsequently, single-robust jamming spectra with SINR and MI criteria were obtained for the fuzzy radar-transmitted waveform and precise target spectrum, as shown in Figures 5 and 7. Finally, double-robust jamming spectra with SINR and MI criteria were designed for the fuzzy radar-transmitted waveform and target spectrum, as shown in Figures 7 and 8.
The power-minimization-based jamming spectra with the SINR criterion are shown in Figure 9. For specific random targets depicted in Figure 6, the jamming power allocation of the optimal jamming spectrum is similar to the robust jamming spectra. Therefore, we only discussed the power allocation of the optimal jamming spectrum.

As can be seen from the top panel of Figure 9, the power allocation of the optimal jamming spectrum is mainly concentrated at the normalized frequencies of $-0.2,0$, and 0.4 , and the spectral amplitude at 0.4 is larger than the spectral amplitude at -0.2 . Note that the target spectral amplitudes at -0.2 and $0.4^{\circ}$ are consistent, as shown in Figure 6. Consequently, we concluded that the jamming spectrum is directly related to the radar-transmitted waveform. Similarly, the middle panel of Figure 9 shows that the spectral amplitude of the optimal jamming spectrum is quite low at a normalized frequency of 0.2 . Therefore, it is inferred that the jamming spectrum is directly related to the target spectrum owing to the tendency of random target 2 , as illustrated in Figure 6.

Figure 10 shows the power-minimization-based jamming spectra with the MI criterion for different random targets. Similar to the discussion above, we observed that the jamming spectra are related to the radar-transmitted waveform and target spectrum. In addition, the power allocations of optimal, single-robust, and double-robust jamming spectra for a fixed spectrum of the target are similar.

The power allocation of the jamming waveform with the SINR and MI criteria is different. The simulation results demonstrate that the power allocation of the jamming spectrum with the SINR criterion is more concentrated in the narrow frequency band near the frequency point of 0.4 , as shown in the top panel of Figure 9, and the power allocation of the jamming spectrum with the MI criterion is more dispersed, as depicted in the top panel of Figure 10. Power-minimization-based jamming waveform design methods lead to different power allocation strategies for different criteria, which provide guidance for power allocation for the power-saving performance of smart jammers in the presence of finite-duration random target. 
TABLE 1: Simulation parameters.

\begin{tabular}{lccc}
\hline Parameter & Value & Parameter & Value \\
\hline$\gamma_{\text {SINR }}$ & $10-50$ & $S_{\mathrm{nn}}(f)$ & $1 \mathrm{w}$ \\
$\gamma_{\mathrm{MI}}$ & $10-50$ & Ty & $1 \mathrm{~s}$ \\
$\Delta f$ & 0.0039 & BW & 256 \\
\hline
\end{tabular}

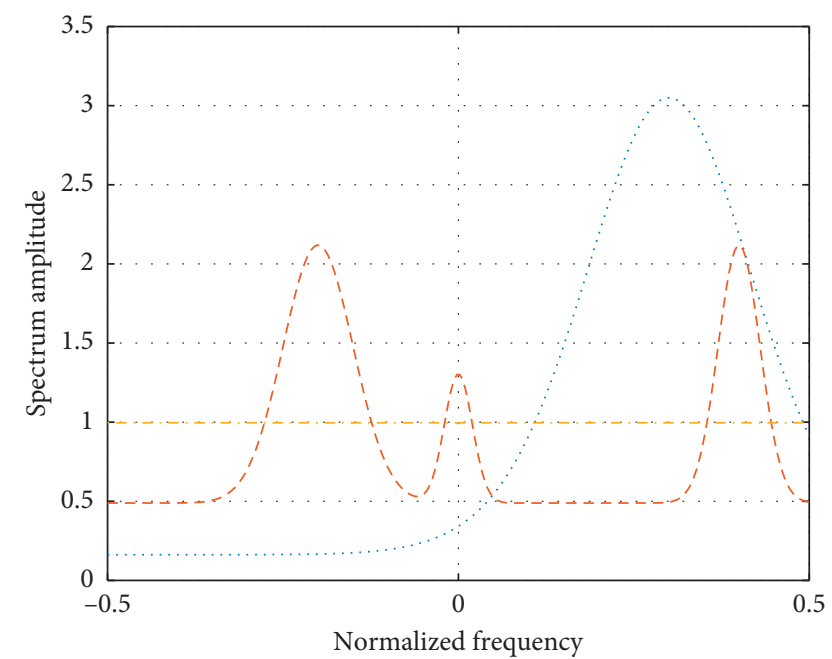

Radar-transmitted waveform

The random target 1

-. - Noise PSD

FIGURE 5: Prior information in the environment.

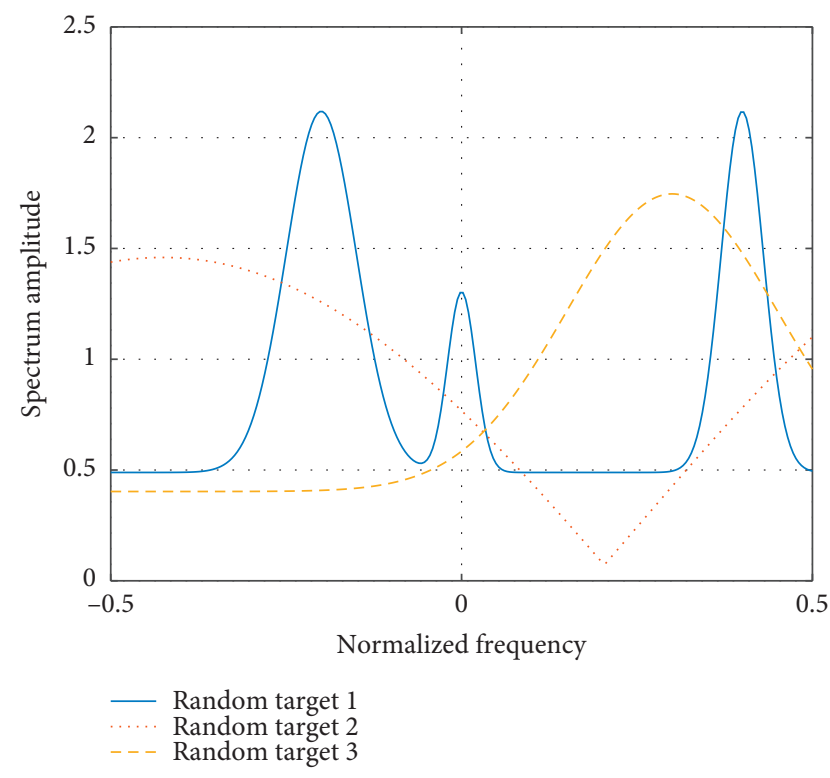

FIgURE 6: Finite-duration random target.

5.3. Power-Saving Performance. In this subsection, the power-saving performance of smart jammers with different algorithms is discussed. The total jamming power versus the SINR and MI thresholds is depicted in Figure 11. As we increase the value of SINR and MI threshold, we observe that

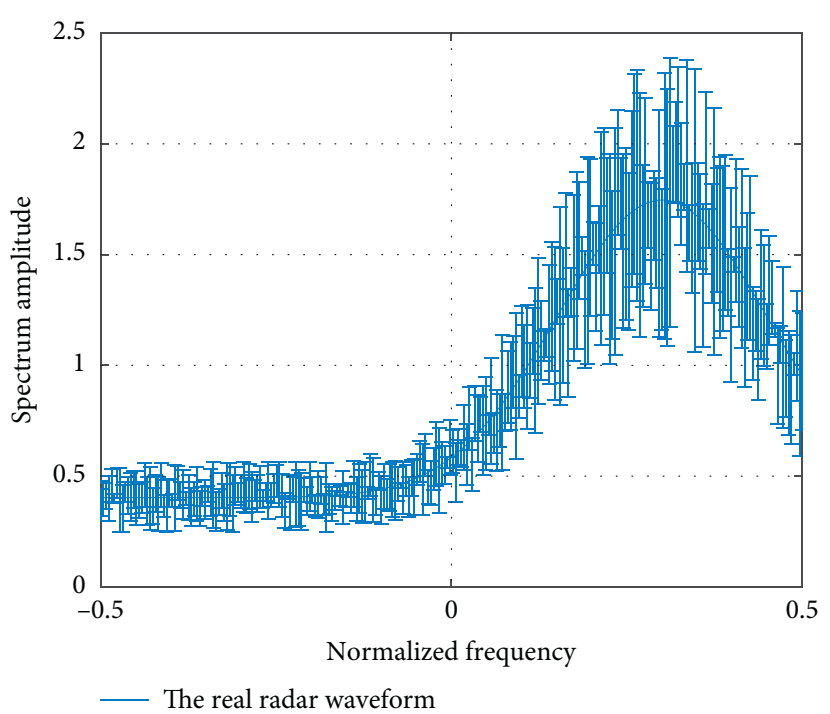

Figure 7: Bounded radar waveform spectrum samples.

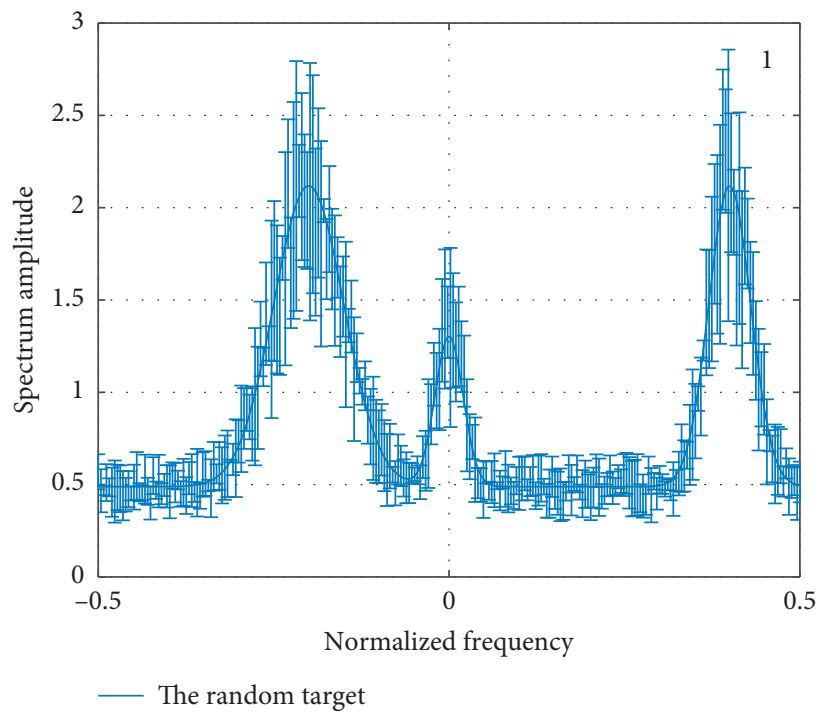

Figure 8: Bounded target spectrum samples.

the power consumption of the smart jammer is greatly decreased, as shown in Figure 11. The decline is owing to less jamming power needed to weaken the detection or parameter estimation performance of the radar system as the specified SINR or MI threshold increases. The power-saving performances of smart jammers at a particular SINR or MI threshold with different algorithms will be discussed later.

Figure 12 illustrates the comparison of the total jamming power employing different algorithms, where Monte Carlo simulations with 100 independent trials are conducted to achieve an average performance. Figure 12(a) shows the jamming power consumption achieved by the SINR limitation. Figure 12(b) illustrates the power consumption of the jamming spectrum for a predetermined MI threshold. The aim of Figure 12 is to prove that the presented algorithms are able to enhance the power-saving performance of smart jammers. 


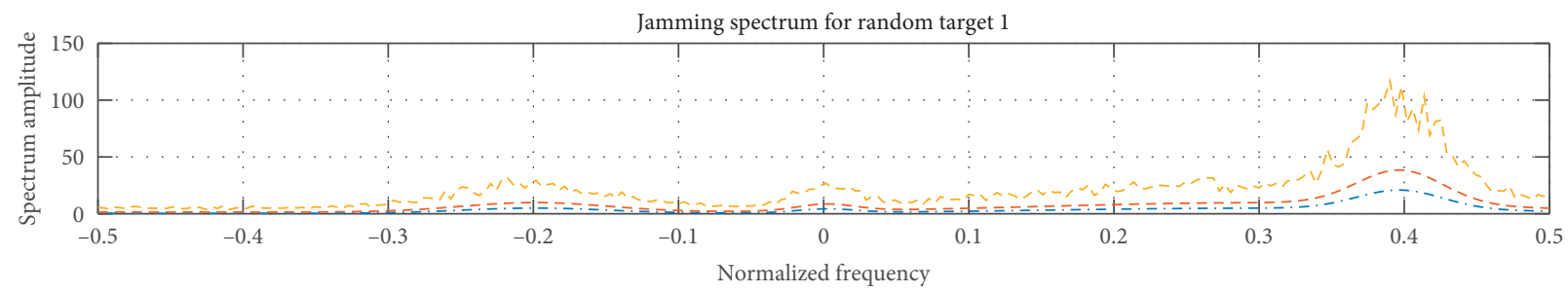

. -. - Optimal jamming waveform for SINR

- - - Single-robust jamming waveform for SINR

- - - Double-robust jamming waveform for SINR

(a)

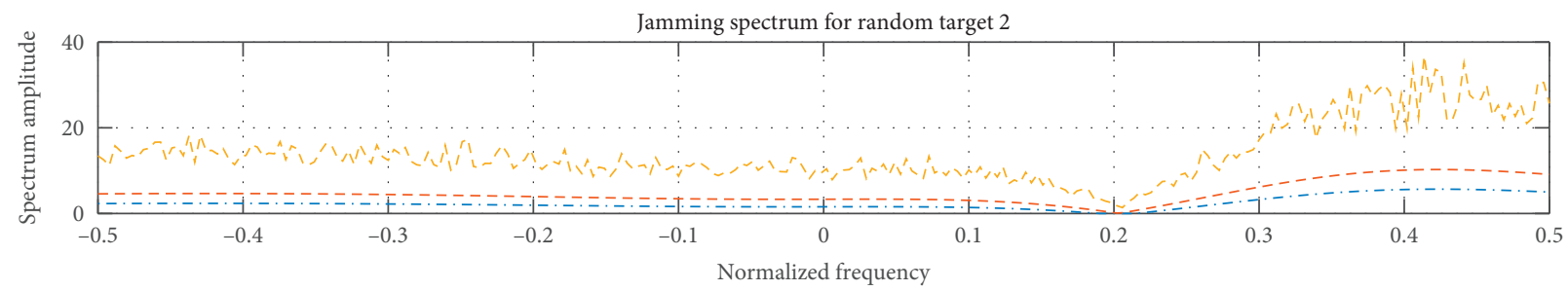

- . - Optimal jamming waveform for SINR

- - - Single-robust jamming waveform for SINR

- - - Double-robust jamming waveform for SINR

(b)

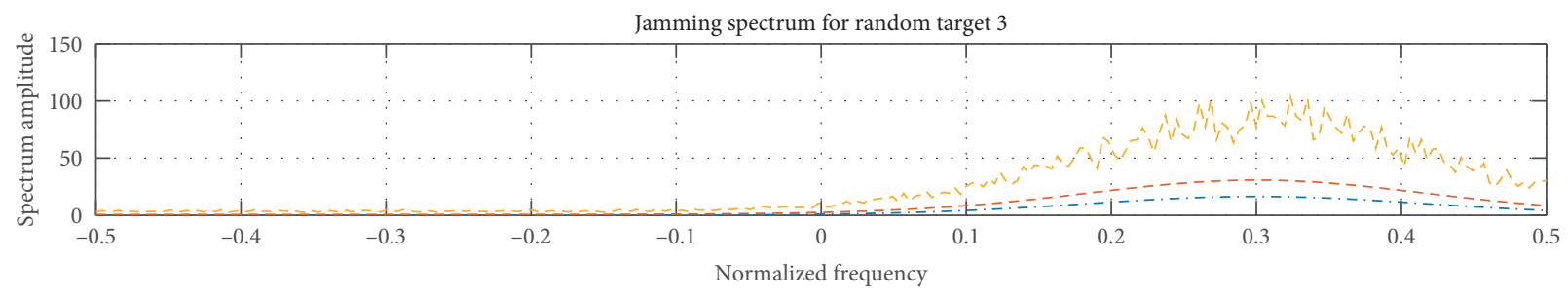

-.- Optimal jamming waveform for SINR

- - - Single-robust jamming waveform for SINR

Double-robust jamming waveform for SINR

(c)

FIGURE 9: Jamming spectra for finite-duration random target with the SINR criterion.

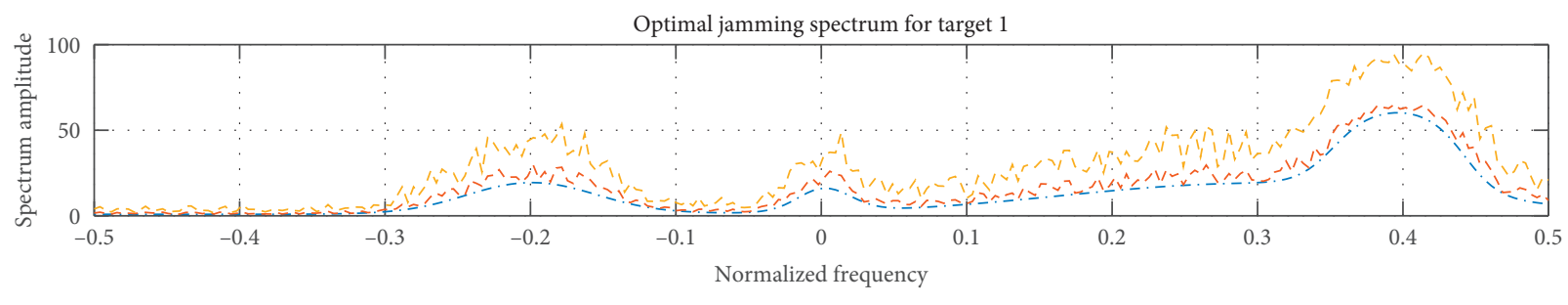

- - - Optimal jamming waveform for MI

- - - Single-robust jamming waveform for MI

- - - Double-robust jamming waveform for MI

(a)

Figure 10: Continued. 


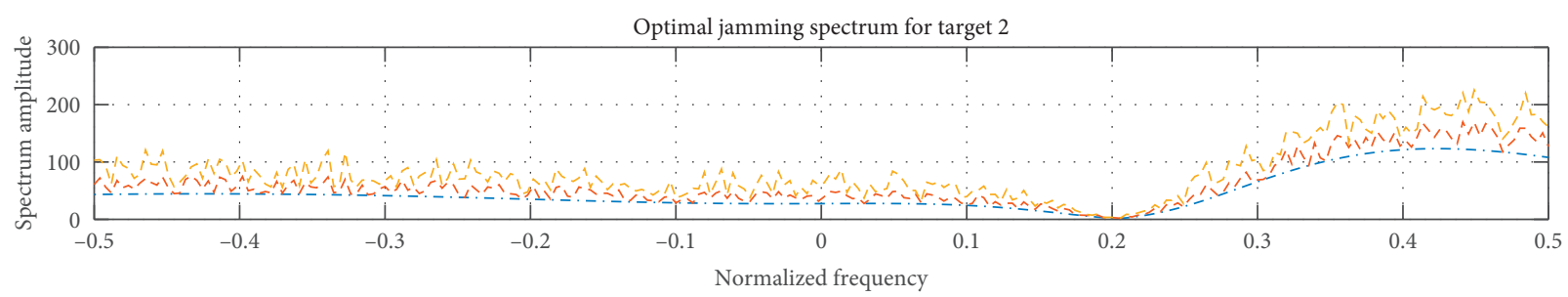

. -. - Optimal jamming waveform for MI

- - - Single-robust jamming waveform for MI

- - - Double-robust jamming waveform for MI

(b)

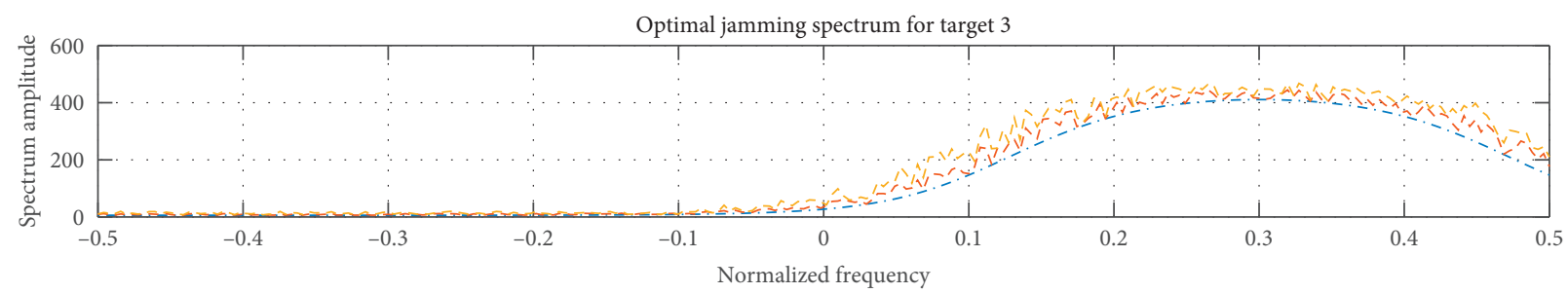

...- Optimal jamming waveform for MI

- - - Single-robust jamming waveform for MI

- - - Double-robust jamming waveform for MI

(c)

FIGURE 10: Jamming spectra for finite-duration random target with the MI criterion.
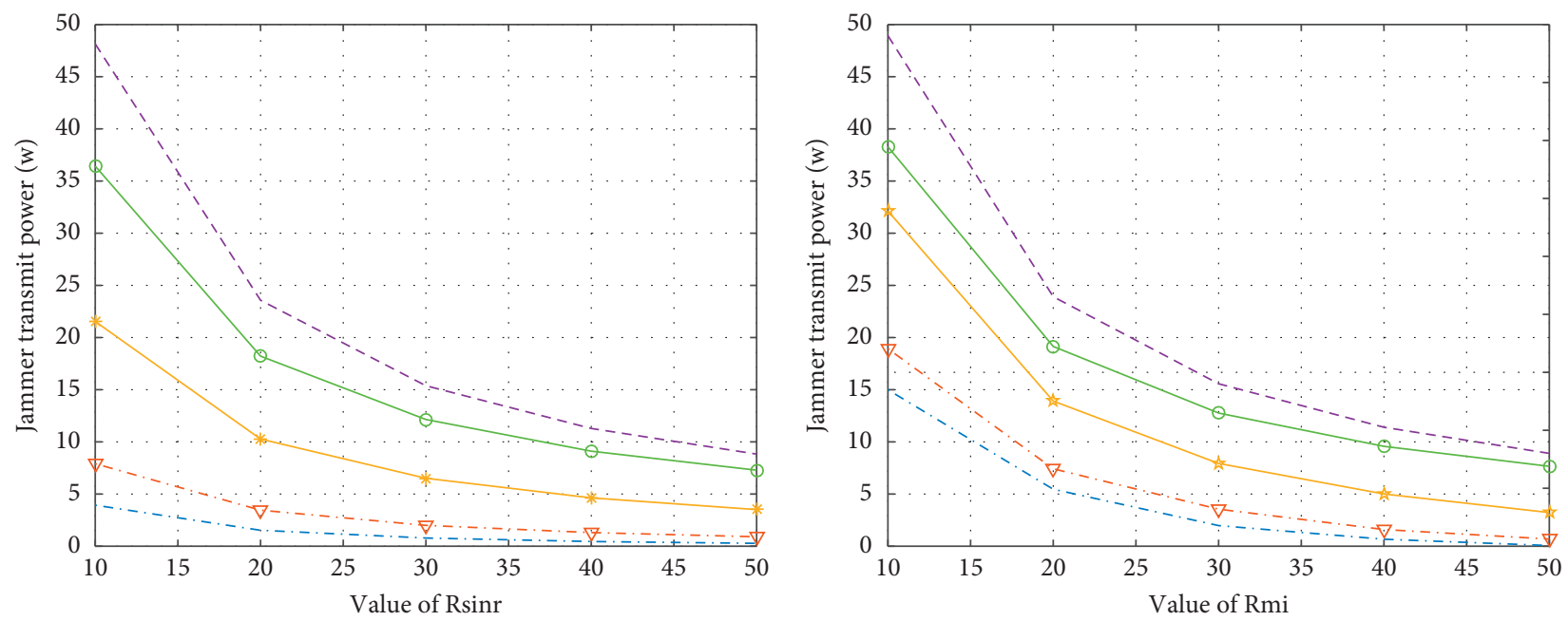

...- The power of optimal jamming

$-\nabla^{-}$The power of single-robust jamming

* - The power of double-robust jamming

-- - The power of wideband jamming

$\multimap$ The power of LFM jamming

(a)

-.- The power of optimal jamming

$\nabla^{-}$The power of single-robust jamming

* The power of double-robust jamming

- - - The power of wideband jamming

- The power of LFM jamming

(b)

FIgURE 11: Trend of jamming power versus the SINR/MI threshold with different algorithms: (a) SINR and (b) MI.

The simulation results show that, as we employ a powerminimization-based optimal jamming waveform design method, a better power-saving performance can be obtained for SINR/MI limitations. This indicates that the jammer transmits the minimum power when assuming that the precise radar and target spectra are known. In addition, we provided simulation data for single- and double-robust, linear frequency modulation (LFM), and wideband jamming spectra. The power consumption of the LFM and wideband jamming spectra is presented as a standard to demonstrate 

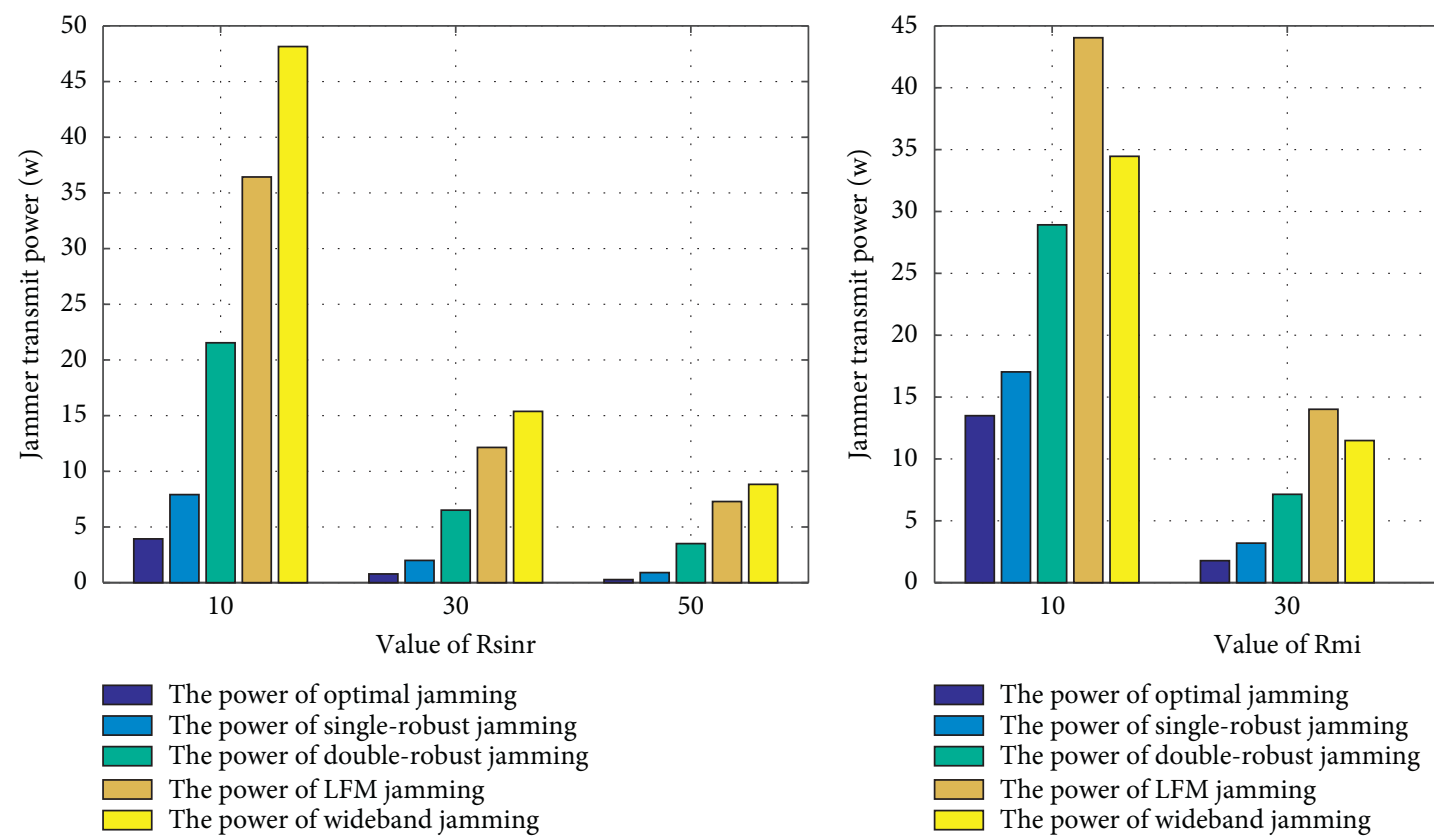

(b)

Figure 12: Comparisons of jamming power with (a) SINR criterion and (b) MI criterion.

the effectiveness of the power-minimization-based jamming waveform design. As shown in Figure 12, LFM and wideband jamming spectra lead to poor power-saving performance because the two strategies lack the prior information of the radar and random target. Moreover, it can be noted that the power-saving performances of the single- and double-robust jamming spectra are second only to the optimal jamming spectrum, which is owing to the fact that power-minimization-based robust jamming waveform design methods have less prior information of radar and target spectra. In addition, the precise prior information of the target cannot be obtained by a smart jammer when employing a double-robust jamming waveform design strategy, which causes the power-saving performance of the single-robust jamming spectrum to outperform the spectrum when employing the double-robust jamming strategy. In summary, this result demonstrates that the double-robust jamming spectrum effectively bounds the worst powersaving performance of the jammer over the uncertainty classes. That is, if the double-robust jamming spectrum is utilized, the power-saving performance will not be worse than this bound.

\section{Conclusions}

In this study, power-minimization-based jamming waveform design methods for finite-duration random targets with SINR and MI criteria were investigated. When employing this optimization strategy, the power-saving performance of the smart jammer is advanced, whereas the detection performance and parameter estimation performance of the radar system are enforced to be below a predetermined level. First, the optimal jamming spectrum was designed assuming that the precise radar and target spectra are known. Subsequently, the uncertainty range of the radar-transmitted waveform was considered, and the single-robust jamming waveform in the local-worst case was designed. Accordingly, the double-robust jamming spectrum was investigated, which bounded the worst powersaving performance of the jammer. The results show that the designed jamming spectra are applicable to finite-duration random targets, whereas the power-saving performance of the jammer system can be optimized in different situations.

\section{Appendix}

\section{$\mathbf{A}$}

The objective function is generated by employing the Lagrangian multiplier technique.

$$
L(\lambda, J(f))=\int_{\mathrm{BW}} J(f) \mathrm{d} f-\lambda\left(\int_{\mathrm{BW}} \frac{\sigma_{H}^{2}(f)|X(f)|^{2}}{J(f)+|X(f)|^{2} S_{\mathrm{cc}}(f)+S_{\mathrm{nn}}(f)} \mathrm{d} f-\gamma_{\mathrm{SINR}}\right),
$$


where $\lambda$ is the Lagrange multiplier. The formula can boil down to $L(J(f))$, which can be denoted as follows:

$$
L(J(f))=J(f)-\lambda \frac{\sigma_{H}^{2}(f)|X(f)|^{2}}{J(f)+|X(f)|^{2} S_{\mathrm{cc}}(f)+S_{\mathrm{nn}}(f)} .
$$

Because the second-order derivative test of $L(J(f))$ with regard to the jamming spectrum $J(f)$ is less than 0 , the analytical solution of $J(f)$ can be derived by letting $L^{\prime}(J(f))=0$.

The optimal jamming waveform can be mathematically represented as follows:

$$
J(f)=\max \left[0,|X(f)| \sqrt{\sigma_{H}^{2}(f)}\left(A-\frac{S_{\mathrm{cc}}(f)|X(f)|^{2}+S_{\mathrm{nn}}(f)}{|X(f)| \sqrt{\sigma_{H}^{2}(f)}}\right)\right],
$$

where $A$ is a constant that can be determined by the SINR

threshold constraint.

$$
\int_{\mathrm{BW}} \frac{\sigma_{H}^{2}(f)|X(f)|^{2}}{\max [0, B(f)(A-D(f))]+S_{\mathrm{cc}}(f)|X(f)|^{2}+S_{\mathrm{nn}}(f)} \mathrm{d} f \leq \gamma_{\mathrm{SINR}} .
$$

\section{B}

The objective function can be generated by employing the Lagrangian multiplier technique.

$$
K\left(\xi, J^{*}(f)\right)=\int_{\mathrm{BW}} J^{*}(f) \mathrm{d} f-\xi\left(\operatorname{Ty} \int_{\mathrm{BW}} \ln \left(1+\frac{\sigma_{H}^{2}(f)|X(f)|^{2}}{\mathrm{Ty}\left(J^{*}(f)+S_{\mathrm{cc}}(f)|X(f)|^{2}+S_{\mathrm{nn}}(f)\right)}\right) \mathrm{d} f-\gamma_{\mathrm{MI}}\right),
$$

where $\xi$ is the Lagrange multiplier. Obtaining the derivative of $K\left(\xi, J^{*}(f)\right)$ with respect to $J^{*}(f)$ and setting it to zero, the analytical solution can be derived as follows:

$$
\begin{aligned}
& J^{*}(f)=\max \left\{0,-Z(f)+\sqrt{Z^{2}(f)+R(f)\left[A^{*}-C(f)\right]}\right\} \\
& Z(f)=V(f)+\frac{|H(f)|^{2}|X(f)|^{2}}{T_{y}} \\
& R(f)=\frac{\sigma_{H}^{2}(f)|X(f)|^{2}}{T_{y}}, \\
& C(f)=\frac{\left(V^{2}(f)+\sigma_{H}^{2}(f)|X(f)|^{2} V(f)\right) / \mathrm{Ty}}{\left(\sigma_{H}^{2}(f)|X(f)|^{2}\right) / \mathrm{Ty}}
\end{aligned}
$$

$\mathrm{A}^{*}$ is a constant that can be defined by the MI threshold constraint. And $V(f)=S_{\mathrm{cc}}(f)|X(f)|^{2}+S_{\mathrm{nn}}(f)$.

$$
\operatorname{Ty} \int_{\mathrm{BW}} \ln \left(1+\frac{\sigma_{H}^{2}(f)|X(f)|^{2}}{\operatorname{Ty}\left(J^{*}(f)+V(f)\right)}\right) \mathrm{d} f \leq \gamma_{\mathrm{MI}} .
$$

To obtain a more intuitive expression, a jamming result can be derived by employing a first-order Taylor series approximation on (B.2).

$$
J^{*}(f)=\max \left[0, G(f)\left(A^{*}-C(f)\right)\right],
$$

where

$$
G(f)=\frac{\left(\sigma_{H}^{2}(f)|X(f)|^{2}\right) / \mathrm{Ty}}{\left(2 V(f)+\sigma_{H}^{2}(f)|X(f)|^{2}\right) / \mathrm{Ty}} .
$$

\section{Data Availability}

The data used to support the findings of this study are included within the article.

\section{Conflicts of Interest}

The authors declare that they have no conflicts of interest regarding the publication of this paper.

\section{Acknowledgments}

This work was supported by the Excellent Going Abroad Experts' Training Program in Hebei Province. 


\section{References}

[1] W. Yuxi, H. Guoce, and L. I. Wei, "Waveform design for radar and extended target in the environment of electronic warfare," Journal of Systems Engineering and Electronics, vol. 29, pp. 48-57, 2018.

[2] R. A. Romero and N. A. Goodman, "Waveform design in signal-dependent interference and application to target recognition with multiple transmissions," IET Radar, Sonar \& Navigation, vol. 3, no. 4, pp. 328-340, 2009.

[3] R. A. Romero, J. Bae, and N. A. Goodman, "Theory and application of SNR and mutual information matched illumination waveforms," IEEE Transactions on Aerospace and Electronic Systems, vol. 47, no. 2, pp. 912-927, 2011.

[4] A. Leshem, O. Naparstek, and A. Nehorai, "Information theoretic adaptive radar waveform design for multiple extended targets," IEEE Journal of Selected Topics in Signal Processing, vol. 1, no. 1, pp. 42-55, 2007.

[5] F. Ahmad and M. G. Amin, "Matched-illumination waveform design for a multistatic through-the-wall radar system," IEEE Journal of Selected Topics in Signal Processing, vol. 4, no. 1, pp. 177-186, 2010.

[6] B. Tang, J. Tang, and Y. Peng, "MIMO radar waveform design in colored noise based on information theory," IEEE Transactions on Signal Processing, vol. 58, pp. 4684-4697, 2010.

[7] C. Shi, F. Wang, S. Salous, and J. Zhou, "Low probability of intercept-based optimal OFDM waveform design strategy for an integrated radar and communications system," IEEE Access, vol. 6, pp. 57689-57699, 2018.

[8] X. Cheng, A. Aubry, D. Ciuonzo, A. De Maio, and X. Wang, "Robust waveform and filter bank design of polarimetric radar," IEEE Transactions on Aerospace and Electronic Systems, vol. 53, no. 1, pp. 370-384, 2017.

[9] A. Abdalla, W.-Q. Wang, Z. Yuan, S. Mohamed, and T. Bin, "Subarray-based FDA radar to counteract deceptive ECM signals," EURASIP Journal on Advances in Signal Processing, vol. 2016, no. 1, p. 104, 2016.

[10] D. Ciuonzo, A. De Maio, G. Foglia, and M. Piezzo, "Intrapulse radar-embedded communications via multiobjective optimization," IEEE Transactions on Aerospace and Electronic Systems, vol. 51, no. 4, pp. 2960-2974, 2015.

[11] L. Bao, C. Wang, and X. Li, "Radar anti-jamming technology based on waveform watermarking," in Proceedings of the 2018 IEEE 3rd Advanced Information Technology, Electronic and Automation Control Conference (IAEAC), pp. 1860-1865, Chongqing, China, October 2018.

[12] H. Jung, B. V. Nguyen, I. Song, and K. Kim, "Design of antijamming waveforms for time-hopping spread spectrum systems in tone jamming environments," IEEE Transactions on Vehicular Technology, vol. 69, no. 1, pp. 728-737, 2020.

[13] K. Zhou, D. Li, Y. Su, and T. Liu, "Joint design of transmit waveform and mismatch filter in the presence of interrupted sampling repeater jamming," IEEE Signal Processing Letters, vol. 27, pp. 1610-1614, 2020.

[14] L. L. Wang, H. Q. Wang, and Y. Q. Cheng, "A novel SINR and mutual information based radar jamming technique," Journal of Central South University, vol. 20, no. 12, pp. 3471-3480, 2013.

[15] L. Wang, H. Wang, K. K. Wong, and P. V. Brennan, "Minimax robust jamming techniques based on signal-to-interferenceplus-noise ratio and mutual information criteria," IET Communications, vol. 8, no. 10, pp. 1859-1867, 2014.

[16] L. Wang, L. Wang, Y. Zeng, and M. Wang, "Jamming power allocation strategy for MIMO radar based on MMSE and mutual information," IET Radar, Sonar \& Navigation, vol. 11, no. 7, pp. 1081-1089, 2017.

[17] C. G. Shi, F. Wang, S. Salous, and J. J. Zhou, "Adaptive jamming waveform design for distributed multiple-radar architectures based on low probability of intercept," Radio Science, vol. 54, no. 1, pp. 72-90, 2019.

[18] Y. Yang and R. S. Blum, "Minimax robust MIMO radar waveform design," IEEE Journal of Selected Topics in Signal Processing, vol. 1, no. 1, pp. 147-155, 2007.

[19] E. Grossi, M. Lops, and L. Venturino, "Robust waveform design for MIMO radars," IEEE Transactions on Signal Processing, vol. 59, no. 7, pp. 3262-3271, 2011.

[20] W. Zhu and J. Tang, "Robust design of transmit waveform and receive filter for colocated MIMO radar," IEEE Signal Processing Letters, vol. 22, no. 11, pp. 2112-2116, 2015.

[21] B. Wang, X. Chen, F. Xin, and X. Song, "MI-based robust waveform design in radar and jammer games," Complexity, vol. 2019, Article ID 4057849, 14 pages, 2019.

[22] C. Shi, F. Wang, M. Sellathurai, J. Zhou, and S. Salous, "Power minimization-based robust OFDM radar waveform design for radar and communication systems in coexistence," IEEE Transactions on Signal Processing, vol. 66, no. 5, pp. 13161330, 2018.

[23] J. Gao, R. Wu, and J. Zhang, "An adaptive multi-target jamming waveform design based on power minimization," Entropy, vol. 22, no. 5, p. 508, 2020. 